\title{
Pattern and position of portal vein formation in a Kenyan population
}

\author{
Abstract \\ Background: Knowledge of the variant anatomy of the extra hepatic portal vein is \\ important for both surgeons and radiologists.
}

Objectives: To describe the pattern and position of formation of the portal vein in a Kenyan population.

Materials and Methods: The study was carried out on 42 cadavers during routine dissection sessions in the Department of Human Anatomy, University of Nairobi. The pattern of PV formation and its location relative to the neck of the pancreas were identified and photographs taken. The pattern of PV formation was classified thus: Type I: PV formed by the confluence of SMV and SV; Type II: PV formed by the confluence of SMV, IMV and SV. Type I was further reclassified according to the termination of IMV as Type Ia: IMV terminate into SV and Type Ib: IMV terminate into the SMV.

Results: Type I pattern of PV formation was observed in 31 cases $(73.8 \%)$ while a Type II pattern was observed in 11 cases $(26.2 \%)$. Of the Type I pattern of PV formation, Type Ia and Type IIb occurred in 15 cases $(48.4 \%)$ and 16 cases $(51.6 \%)$ respectively. The PV formed inferior to the neck of the pancreas in 9 cases $(21.4 \%)$, posterior to it in 25 cases $(59.5 \%)$ and superior to it in 8 cases $(19 \%)$.

Conclusion: The PV displays variations both its pattern of formation and location. This should be taken into consideration during pancreatico-duodenal surgeries to avoid inadvertent injury to the PV and in the interpretation of abdominal radiographs.
Volume 3 Issue 3 - 2017

\author{
Munguti J,' Sammy M, ${ }^{2}$ Cheruiyot I,' Kariuki \\ $B,{ }^{\prime}$ Olabu $B^{\prime}$ \\ 'Department of Human Anatomy, University of Nairobi, Kenya \\ 2Maua Methodist Hospital, Kenya
}

Correspondence: Munguti J, Department of Human Anatomy, University of Nairobi, Kenya, Email donaldjrmh86@gmail.com

Received: December 19, 2016 | Published: March 20, 2017

Keywords: portal vein, pattern, location, kenyan population

Abbreviations: PV, portal vein; SMV, superior mesenteric vein; SV, splenic vein; IMV, inferior mesenteric vein

\section{Introduction}

The portal vein (PV), which provides the bulk of nutritive blood supply to the liver, is formed by the confluence of the superior mesenteric vein (SMV) and splenic vein (SV) posterior to the neck of the pancreas. ${ }^{1}$ It courses superiorly towards the liver and lies as the most posterior of the triad of structures found within the hepatoduodenal ligament. Variant anatomy of the PV origin has been reported in literature albeit with varying figures. ${ }^{2-4}$ Knowledge on the incidence of variations in the formation of the PV is important in performing pancreatic and duodenal surgeries, for radiologists performing radiological procedures and in the interpretation of radiological images of the abdomen.

Majority of the variations in the formation of the PV involve the inferior mesenteric vein (IMV) which is known to have a variant pattern of termination. Classically, the IMV terminates into the SV but has also been found to terminate either into the SMV or at the splenoportal junction. ${ }^{2-7}$ Besides, various case reports have reported the congenital absence of the PV. ${ }^{7-9}$

Even though the $\mathrm{PV}$ is described to originate posterior to the neck of the pancreas, ${ }^{1}$ pre pancreatic origins have been described. ${ }^{10}$ Furthermore, there is barely scientific literature detailing the location of the PV relative to the neck of the pancreas. The aim of this study therefore was to describe variations in the formation of the PV in a Kenyan population.

\section{Methods}

The present study was carried out during routine dissection in the Department of Human Anatomy, University of Nairobi. A total of 42 adult cadavers ( 27 male and 15 female) were included in this study. Cadavers that had features of previous abdominal surgeries or trauma were excluded from the study. After a midline abdominal incision to open the abdomen was made, the SMV, IMV and SV were identified and followed up to the point of PV was formation. Photographs of the variations noted were taken.

The pattern of PV formation was classified thus:

i. Type I: PV formed by the confluence of SMV and SV;

ii. Type II: PV formed by the confluence of SMV, IMV and SV.

Type I was further reclassified according to the termination of IMV as Type Ia, IMV terminate into SV and Type Ib, IMV terminate into the SMV.

This classification has been used by previous authors. ${ }^{3,4}$ The point of PV formation relative to the neck of the pancreas was noted and recorded as: 
a. Inferior to the neck of the pancreas,

b. Posterior to the neck of the pancreas, and

c. Superior to the neck of the pancreas.

\section{Results}

The PV was present in all the 42 cadavers studied. Type I pattern of PV formation was observed in 31 cases $(73.8 \%)$ while a Type II pattern was observed in 11 cases (26.2\%). Of the Type I pattern of PV formation, Type Ia and Type Ib occurred in 15 cases (48.4\%) and 16 cases $(51.6 \%)$ respectively (Table 1$)$.
Table I Pattern of IMV termination

\begin{tabular}{lll}
\hline Point of termination & Frequency & Percentage (\%) \\
\hline Splenic Vein & 15 & 35.7 \\
Splenoportal Junction & 11 & 26.2 \\
Superior Mesenteric Vein & 16 & 38.1 \\
Total & 42 & 100 \\
\hline
\end{tabular}

The PV formed inferior to the neck of the pancreas in 9 cases (21.4\%), posterior to it in 25 cases (59.5\%) and superior to it in 8 cases (19\%) (Figures 1A-1D).


Figure IA Type IA pattern of formation of the portal vein [PV] by union of the superior mesenteric vein [SMV] and the splenic vein [SV]. Note the inferior mesenteric vein $[\mathrm{IMV}]$ joining the splenic vein.

Figure IB Type IB pattern of formation of the portal vein [PV] by the union of the superior mesenteric vein [SMV] and the splenic vein [SV]. The inferior mesenteric vein $[\mathrm{SMV}]$ drains to the superior mesenteric vein.
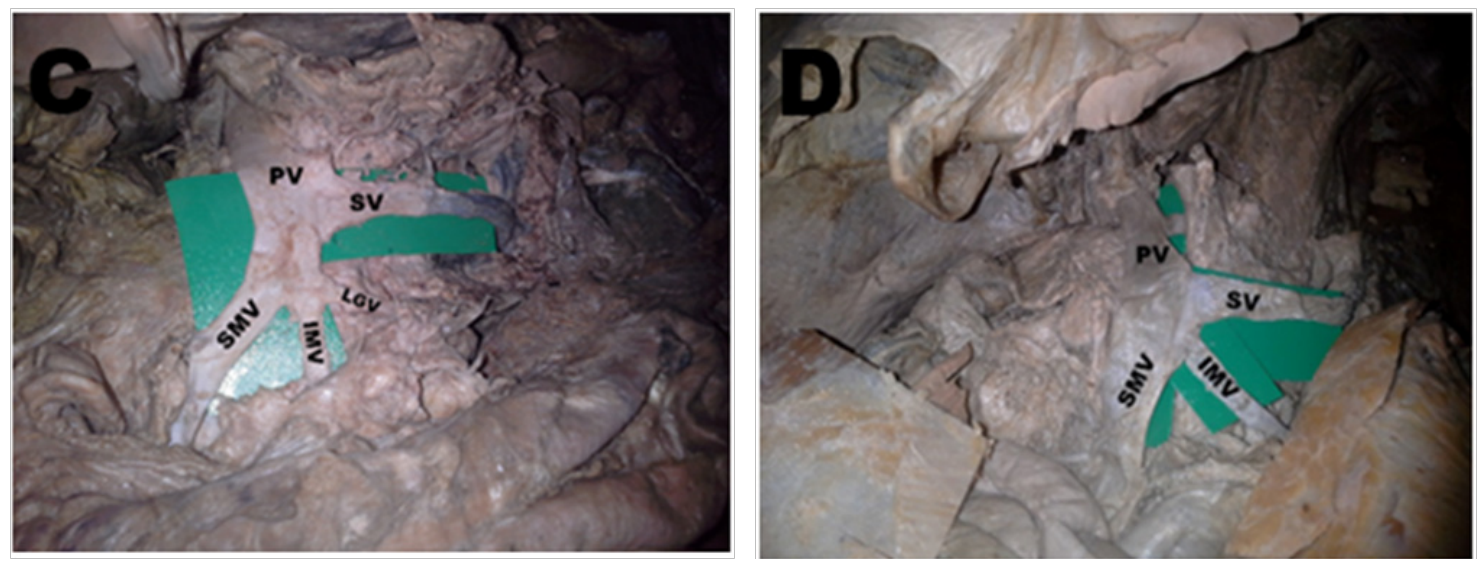

Figure IC Type IB pattern of formation of the portal vein [PV] by the union of the superior mesenteric vein [SMV] and the splenic vein [SV]. The inferior mesenteric vein [SMV] drains to the superior mesenteric vein. Note the left gastroepiploic vein [LGV] joining the superior mesenteric vein together with the inferior mesenteric vein.

Figure ID Type II pattern of formation of the portal vein [PV] by triple confluence of the superior mesenteric vein [SMV], inferior mesenteric vein [IMV] and the splenic vein [SV].

Figure IA-D: Various patterns of portal vein formation.

\section{Discussion}

With advancement of bariatric, hepatobiliary and pancreatic surgeries, knowledge on the variant anatomy of the PV has become of paramount importance. This is because iatrogenic PV injury following pancreatico-duodenectomy may precipitate massive postoperative hemorrhage which may be life threatening. Moreover, the risks of PV injury following blunt or penetrating abdominal trauma are real which necessitates its surgical repair. ${ }^{11}$ 
The current study did not report any congenital absence of the PV underscoring the rarity of the same as previously reported. The prevalence of Type I pattern of formation has been reported to range from $40 \%$ to $84.62 \% .{ }^{2-4}$ The current study's figure of $73.8 \%$ is in agreement with these findings. Majority of these studies were cadaveric and even though they had a varied sample size (range of 30 to 271), the variable pattern of PV formation cannot be overlooked.

The IMV pattern of termination is known to be the most varied of the major enteric veins and has been known to form unusual portosystemic venous shunts with the left internal iliac vein. ${ }^{12}$ In the present study, its classical termination into the SV occurred in $35.7 \%$. Previous reports have put this figure to be as low as $18 \%$ and as high as $58 \%{ }^{3-5}$ About a quarter of IMVs in the current study terminated by joining the PV at the splenoportal junction unlike in other reports where it terminated in a Type II pattern with a prevalence of $47 \% .^{3}$ Two studies reported this figure at 10 and $15 \% \%$ respectively. ${ }^{2,4}$ Anomalous formations of the PV previously reported include: preduodenal PV with several accessory portal branches flowing directly in to hepatic segments; ${ }^{13}$ multiple SMVs joining with the SV to form the PV; ${ }^{14}$ multiple jejunal veins, SMV and SV all joining together to form the PV; ${ }^{15}$ preduodenal $\mathrm{SMV} ;{ }^{16}$ and the left gastric vein joining the SMV before it joins the SV to form the PV. ${ }^{17}$ All this variability in the formation of the PV should be borne in mind whenever performing intra-abdominal surgeries or interpreting radiological images of the abdomen.

Various attempts have been made to describe the point of formation of the PV relative to the neck of the pancreas or the lumbar vertebrae. ${ }^{11}$ Awareness of the variant anatomy involved is beneficial for both surgeons and interventional radiologists. In the current study, it was observed that the point of PV formation was always posterior to the neck of the pancreas. Prepancreatic PV formation has been reported previously and its occurrence should be expected albeit rarely. ${ }^{10}$ Even though a majority of the PVs in the current study formed posterior to the neck of the pancreas, $40 \%$ of its formation occurred either superior to or inferior to the pancreatic neck. Pancreatitis occasionally leads to vascular complications including venous occlusion. The most vulnerable structure is the SV but the variable point of PV formation, as observed in the current study, may explain the varied severity of the clinical picture seen in vasculitis secondary to pancreatitis.

\section{Conclusion}

The PV displays variations both its pattern of formation and location. This should therefore be taken into consideration during pancreatico-duodenal surgeries and in the interpretation of abdominal radiographs to avoid inadvertent injury to the PV.

\section{Acknowledgements}

None.

\section{Conflict of interest}

Author declares that there is no conflict of interest.

\section{References}

1. Abdel Misih SRZ, Bloomston M. Liver anatomy. Surg Clin North Am. 2010;90(4):643-653.

2. Kaur H, Singh M, Bajaj A. A cadaveric study of morphology of portal vein with its clinical importance. Med J DY Patil Univ. 2016;9(3):336-340.

3. Raut R, Bahetee B. Study of variations in the formation of portal vein. Sch J App Med Sci. 2015;3(3E):1370-1375.

4. Chaijaroonkhanarak W, Woraputtaporn W, Amarttayakong P, et al. Length, diameter and variations in types of portal vein formations in northeastern thais. Srinagarind Med J SMJ. 2011;25(4):323-327.

5. Khamanarong K, Woraputtaporn W, Amarttayakong $\mathrm{P}$, et al. Classification of portal vein tributaries in Thai cadavers including a new type V. Surg Radiol Anat. 2015;38(6):735-739.

6. Sztika D, Zăhoi DE, Motoc A, et al. Anatomical variations of the hepatic portal vein associated with incomplete celiac trunk. Rom J Morphol Embrol. 2011;52(2):695-698.

7. $\mathrm{Hu}$ GH, Shen LG, Yang J, et al. Insight into congenital absence of the portal vein: Is it rare? World J Gastroenterol. 2008;14(39):5969-5979.

8. Asran MK, Loyer EM, Kaur H, et al. Case 177: Congenital absence of the portal vein with hepatic adenomatosis. Radiology. 2012;262(1):364-367.

9. Gadodia A, Sharma R, Kandpal H, et al. Congenital absence of portal vein with large inferior mesenteric-caval shunt. Trop Gastroenterol. 2011;32(3):223-226.

10. Chitra P, Maheshwari K, Anadhi V. Prepancreatic formation of portal vein associated with pre pancreatic superior mesenteric artery and splenic vein. International Journal of Anatomical variations. 2014; 7:35-36.

11. Mantovani M, Leal RF, Fontelles MJ. Anatomic study of portal vein: trans pancreatic vessels injuries approach. Acta Cir Bras. 2002;17(4):225-231.

12. Otake M, Kobayashi Y, Hashimoto D, et al. An inferior mesentericcaval shunt via the enteric iliac vein with portosystemic encephalopathy. Intern Med. 2001;40(9):887-890.

13. Kamitani T, Tajima T, Kakihara D, et al. Anomalous portal branches associated with polysplenia syndrome: imaging findings. CMIG Extra Cases. 2004;28(2):15-19.

14. Knight HO. An anomalous portal vein with its surgical dangers. Ann Surg. 1921;74(6):697-699.

15. Nayak SB, Aithal AP, Melanie RD, et al. Unusual jejunal tributaries of the splenic vein and their surgical importance: a case report. $O A$ Case Reports. 2013;2(4):36.

16. Höing K, Ringe KI, Bektas H, et al. Preduodenal superior mesenteric vein and Whipple procedure with vascular reconstruction-A case report. Int J Surg Case Rep. 2015;10:107-110.

17. Gorantla VR, Potu BK, Pulakunta T, et al. Anomalous formation of the portal vein: a case report. J Vasc Bras. 2007;6(4):399-401. 